\title{
Dehmel, Niels (2020): Wege aus dem Wahlrechtsdilemma. Eine komparative Analyse ausgewählter Reformen für das deutsche Wahlsystem
}

\author{
Baden-Baden: Nomos. 875 Seiten. 139,00€
}

\section{Joachim Behnke}

Angenommen: 29. Juni 2021 / Online publiziert: 19. Juli 2021

(C) Der/die Autor(en) 2021

Niels Dehmel hat mit seiner über 800 Seiten umfassenden Monografie „Wege aus dem Wahlrechtsdilemma. Eine komparative Analyse ausgewählter Reformen für das deutsche Wahlsystem" einen im wörtlichen Sinn gewichtigen Beitrag für die aktuelle Wahlrechtsdebatte geliefert.

Dehmels Arbeit fokussiert sich auf diejenigen Elemente eines Wahlsystems, welche im Rahmen der aktuellen Wahlsystemdebatte eine gewisse Rolle spielen. Er selbst spricht dabei von einer Eingrenzung seines Gegenstands auf ,strukturerhaltende Reformen der personalisierten Verhältniswahl“", was in der Debatte häufig unter dem Schlagwort des ,minimal-invasiven Eingriffs“ verhandelt wurde. Nach Dehmels Ansicht hat sich der Typus der personalisierten Verhältniswahl für das politische System der Bundesrepublik bewährt. Es geht ihm daher darum, Reformvorschläge zu präsentieren, die die ,Funktions- und Leistungsfähigkeit des existierenden Wahlsystems“ zu steigern vermögen. Reformvorschläge, die systemverändernden Charakter hätten, wie insbesondere die reine Mehrheitswahl, das Grabenwahlrecht oder allgemein alle Vorschläge, die die Komponente der Mehrheitswahl gegenüber dem Status quo deutlich verstärken würden, werden daher nicht behandelt.

Der Hauptteil des Werks, in dem diese verschiedenen strukturerhaltenden Reformansätze abgehandelt werden, befasst sich mit den sieben Themenblöcken der Wahlkreiseinteilung, der Kandidatenaufstellung, der Stimmgebung, der Sperrklauseln, der Überhang- und Ausgleichsmandate, des Wahlalters und der zeitlichen Rahmenbedingungen. Da innerhalb jedes Themenblocks, der sich auf eines dieser Reformelemente bezieht, mehrere Reformvorschläge diskutiert werden, werden insgesamt 24 Reformmöglichkeiten erörtert. Dabei kommen die bekannten und am stärksten diskutierten Vorschläge wie die Verringerung der Anzahl der Wahlkreis-

Joachim Behnke $(\bowtie)$

Zeppelin Universität, Friedrichshafen, Deutschland

E-Mail: joachim.behnke@zu.de 
mandate, die Einführung eines Einstimmensystems und sogenannte Kompensationsmodelle, bei denen Überhangmandate mit Listenmandaten verrechnet werden, vor, aber auch Vorschläge wie Mehrpersonenwahlkreise (in einer bestimmten Form), das sogenannte Kontingent-Verfahren (eine Mischung zwischen starren und losegebundenen Listen) oder die Ersatz- bzw. Nebenstimme, die in der Debatte eher weniger Aufmerksamkeit finden. Nach der Logik einer klassischen vergleichenden Analyse werden alle 24 Reformvorschläge nach bestimmten Kriterien bewertet. Der Kriterienkatalog umfasst die folgenden acht Aspekte: Stimmen-Mandate-Relation (im Wesentlichen die Einhaltung des Proportionalitätserfordernisses zwischen den Parteien, aber auch der unterschiedliche Einfluss auf die Sitzverteilung von Stimmen für im Parlament vertretenen Parteien versus Stimmung für Parteien, die nicht bei der Sitzvergabe berücksichtigt werden), Repräsentation (Vertretung gesellschaftlich relevanter Gruppen), Konzentration, Effektivität (Regierungsstabilität), Partizipation, Zurechenbarkeit von Entscheidungen, Transparenz und Legitimität. Nach jedem Themenblock zu einem Wahlsystemelement gibt es eine ausführliche Bewertung der einzelnen Reformoptionen zu diesem Element sowie eine tabellarische Gesamtübersicht am Ende des Kapitels.

Trotz der umfassenden Behandlung von Reformmöglichkeiten fallen allerdings bestimmte Lücken auf. So geht der Autor davon aus, dass von der Logik eines reinen Proporzes, wie sie im Wahlgesetz von 2013 verwirklicht wird, nicht mehr abgewichen werden kann. Da nach dem Urteil des Bundesverfassungsgerichts von 2012, das von ihm ausführlich diskutiert wird, bis zu 15 unausgeglichene Überhangmandate theoretisch möglich wären (wenn auch nicht unbedingt gut zu begründen), fehlen die Ansätze, die sich diesen Spielraum zunutze machen versuchen. Dass diese eine nicht unerhebliche Rolle spielen, zeigt sich unter anderem am neuen Wahlgesetz von 2020, in dem ja drei unausgeglichene Überhangmandate enthalten sind, nachdem es zuvor sogar weitergehende Forderungen der Union von sieben unausgeglichenen Überhangmandaten gegeben hatte oder im sogenannten Schäuble-Entwurf sogar 15 unausgeglichene Überhangmandate vorgesehen waren. Genauso fehlt eine eigenständige Behandlung sogenannter Kappungsmodelle, bei denen überzählige Direktmandate nicht vergeben werden. Diese Modelle finden lediglich kurz im Rahmen der Diskussion des Kompensationsmodells nach der Logik des Grünenreformvorschlags von 2011 Erwähnung. Eine Behandlung als eigenständiger Reformansatz wäre aber angemessener, da die Kappung ein unabhängiges Element darstellt, das mit der Kompensation verbunden sein kann wie bei dem Grünenvorschlag, oder aber eben gerade nicht, wie z. B. im Reformvorschlag der AfD von 2020. Dieser Ansatz wirft grundsätzliche verfassungs- und gerechtigkeitstheoretische Fragen auf, aber er ist aus diesen Gründen keineswegs von vornherein auszuschließen.

Als Quintessenz seiner vergleichenden Analysen kommt Dehmel am Ende auf zwei Reformvorschläge, die jeweils Verbesserungsvorschläge bezüglich mehrerer Elemente kombinieren. Allerdings stehen diese beiden Ansätze in einem Tradeoff zueinander, man muss sich also für einen der beiden entscheiden. Der erste Ansatz besteht in der ,,verständlichkeitsorientieren personalisierten Verhältniswahl“" (S. 778). Dessen Grundzüge bestehen in der Einführung eines Einstimmensystems mit einer Nebenstimme, die zum Tragen kommt, falls die Partei des mit der Hauptstimme (Erstpräferenz) gewählten Kandidaten an der Fünfprozenthürde 
scheitert. Der zweite Reformansatz, das „,beteiligungsorientierte personalisierte Verhältniswahlsystem“ (S. 778), sieht eine Erhöhung der Partizipationsmöglichkeiten des Wählers durch die Einführung von lose gebundenen Listen vor. Da Dehmel das „Fehlen jeglicher Partizipationschancen abseits des eigentlichen Wahlakts“ als das „wohl größte Defizit“ (S. 783) des aktuellen Systems ansieht, spricht er sich für den zweiten Reformansatz aus. In beiden Reformansätzen sind eine Abschaffung der Grundmandatsklausel und eine Verlängerung der Wahlperiode vorgesehen.

Insgesamt fallen die Reformvorschläge also eher mager aus bzw. - positiv formuliert - schneidet das aktuelle Wahlsystem in seinen Grundzügen aus der Sicht Dehmels so gut ab, dass die meisten Elemente in ihrer bisherigen Form beibehalten werden können. Dabei ist allerdings hervorzuheben, dass seine Reformansätze beide nichts gegen die Vergrößerung des Bundestags unternehmen würden. Dies verwundert insofern, als die Reformdiskussion seit 2013 und noch einmal mit stark erhöhten Nachdruck seit 2017 die Bundestagsvergrößerung als das Übel am Wahlsystem ins Auge nimmt, das es vornehmlich zu bekämpfen gilt. Angesichts einer veränderten und volatilen Parteienstruktur, bei der auch Bundestagsgrößen von über 800 oder sogar 900 Sitzen angesichts aktueller Umfragen nicht mehr völlig unvorstellbar sind, hätte man bei der Synthese durchaus einen Vorschlag erwartet, der dieses Problem zumindest mit in den Fokus nimmt.

Das neue Wahlgesetz von 2020, zu dem Dehmel in seinem Buch noch nicht Stellung nehmen konnte, stellt nur eine Zwischenetappe der Debatte dar, da die Mängel des Systems damit nicht ausgeräumt wurden. Die Debatte wird daher mit Sicherheit in der nächsten Legislaturperiode unvermindert fortgeführt werden. Vor diesem Hintergrund stellen die Einzelanalysen zu den verschiedenen Reformelementen eine konzentrierte Quelle an Information zu diesen dar, die für jedes zukünftige wissenschaftliche Werk in dieser Thematik von großem Wert sein wird. Auch für politische Entscheidungen können die Einzelanalysen zur Bildung eines Rasters für die systematische Untersuchung von Reformvorschlägen herangezogen werden. Damit kann Dehmels Buch nicht nur - wie am Anfang erwähnt - als gewichtiger, sondern auch als wichtiger Beitrag für die Wahlsystemreformdebatte Wirkung entfalten.

Funding Open Access funding enabled and organized by Projekt DEAL.

Open Access Dieser Artikel wird unter der Creative Commons Namensnennung 4.0 International Lizenz veröffentlicht, welche die Nutzung, Vervielfältigung, Bearbeitung, Verbreitung und Wiedergabe in jeglichem Medium und Format erlaubt, sofern Sie den/die ursprünglichen Autor(en) und die Quelle ordnungsgemäß nennen, einen Link zur Creative Commons Lizenz beifügen und angeben, ob Änderungen vorgenommen wurden.

Die in diesem Artikel enthaltenen Bilder und sonstiges Drittmaterial unterliegen ebenfalls der genannten Creative Commons Lizenz, sofern sich aus der Abbildungslegende nichts anderes ergibt. Sofern das betreffende Material nicht unter der genannten Creative Commons Lizenz steht und die betreffende Handlung nicht nach gesetzlichen Vorschriften erlaubt ist, ist für die oben aufgeführten Weiterverwendungen des Materials die Einwilligung des jeweiligen Rechteinhabers einzuholen.

Weitere Details zur Lizenz entnehmen Sie bitte der Lizenzinformation auf http://creativecommons.org/ licenses/by/4.0/deed.de. 